\title{
Prevalence of ventilator-associated pneumonia in a university hospital and prognosis for the patients affected* $^{*}$
}

\author{
MÁRCIO MARTINS DE QUEIROZ GUIMARÃES', JOSÉ RODOLFO ROCCO²
}

\begin{abstract}
Objective: To determine the prevalence of ventilator-associated pneumonia in an intensive care unit, as well as to identify related factors and characterize patient evolution. Methods: This study evaluated 278 patients on mechanical ventilation for more than 24 hours in a university hospital. Results: Ventilator-associated pneumonia developed in 38.1\% of the patients, translating to 35.7 cases/1000 ventilator-days: 45.3\% were caused by gram-negative agents (Pseudomonas aeruginosa accounting for 22\%); and multidrug resistant organisms were identified in 43.4\%. In the ventilator-associated pneumonia group, time on mechanical ventilation, time to mechanical ventilation weaning, hospital stays and intensive care unit stays were all longer $(\mathrm{p}<0.001)$. In addition, atelectasis, acute respiratory distress syndrome, pneumothorax, sinusitis, tracheobronchitis and infection with multidrug resistant organisms were more common in the ventilator-associated pneumonia group ( $p<0.05$ ). Mortality rates in the intensive care unit were comparable to those observed in the hospital infirmary. Associations between ventilator-associated pneumonia and various factors are expressed as odds ratios and $95 \%$ confidence intervals: acute sinusitis (38.8; 3.4-441); > 10 days on mechanical ventilation (7.7; 4.1-14.2); immunosuppression (4.3; 1.314.3); acute respiratory distress syndrome (3.5; 1.4-9.0); atelectasis (3.0; 1.2-7.3); cardiac arrest (0.18; 0.05-0.66); and upper gastrointestinal tract bleeding $(0.07 ; 0.009-0.62)$. The variables found to be associated with in-hospital death were as follows: chronic renal failure $(26.1$; 1.9-350.7); previous intensive care unit admission (15.6; 1.6-152.0); simplified acute physiologic score 11 > 50 (11.9; 3.4-42.0); and age > 55 years (4.4; 1.6-12.3). Conclusion: Ventilator-associated pneumonia increased the time on mechanical ventilation and the number of complications, as well as the length of intensive care unit and hospital stays, but did not affect mortality rates.
\end{abstract}

Keywords: Bacterial pneumonia; Hospital infection; Intensive care units; Prognosis; Prospective studies; Intensive care medicine

\footnotetext{
* Study carried out in the Intensive Care Unit, Center for Surgery and Internal Medicine, Clementino Fraga Filho University Hospital, Universidade Federal do Rio de Janeiro (UFRJ, Federal University of Rio de Janeiro) - Rio de Janeiro, Rio de Janeiro, Brazil

1. Masters in Medicine from the Universidade Federal do Rio de Janeiro (UFRJ, Federal University of Rio de Janeiro) - Rio de Janeiro, Rio de Janeiro, Brazil

2. Adjunct Professor of Basic Clinical Medicine at the Universidade Federal do Rio de Janeiro (UFRJ, Federal University of Rio de Janeiro) - Rio de Janeiro, Rio de Janeiro, Brazil

Correspondence to: Márcio Martins de Queiroz Guimarães. Rua Visconde de Pirajá, 295/702, Ipanema - CEP 22410-001, Rio de Janeiro, RJ, Brasil. Phone: 5521 2267-2428. E-mail: marcio@rj.net

Submitted: 29 April 2005. Accepted, after review: 30 November 2005.
} 


\section{INTRODUCTION}

Pneumonia is the second leading nosocomial infection ${ }^{(1-2)}$ and presents high mortality rates. ${ }^{(3)}$ Ventilator-associated pneumonia (VAP) is the leading infection ${ }^{(4)}$ in intensive care units (ICUs). The incidence ranges from $9 \%$ to $68 \%$, depending on the diagnostic method used and on the population studied. 1ts lethality is high, ranging from 33\% to $71 \%$, and the case fatality rate can reach up to $55 \% .{ }^{(5)}$ Of all cases of hospital-acquired pneumonia, $86 \%$ are associated with mechanical ventilation (MV). ${ }^{(2)}$ However, only $9 \%$ to $27 \%$ of mechanically ventilated patients develop pneumonia. ${ }^{(6-7)}$ The prevalence reported is 20.5 to 34.4 cases/1000 MV days, compared with 3.2 cases/1000 days for patients not on ventilation. ${ }^{(8-9)}$ The proportion of intubated patients who develop pneumonia varies from $10 \%$ to $50 \%$, with an approximate risk of $1 \%$ to $3 \%$ per day of endotracheal intubation. ${ }^{(10-11)}$

The objective of the present study was to determine the prevalence of VAP in mechanically ventilated patients, as well as to identify factors related to VAP and to determine its in-hospital prognostic.

\section{METHODS}

The study was carried out in the ICU of the Clementino Fraga Filho University Hospital of the Federal University of Rio de Janeiro from September of 1999 to February of 2001. This is a ten-bed ICU for adult patients, with an annual admission rate of approximately 600 patients. Patients who required MV for at least $24 \mathrm{~h}$ (consecutively) after admission were studied prospectively. Data regarding the following variables were collected within the first $24 \mathrm{~h}$ after admission: comorbidities and acute diseases; physical examination - arterial blood pressure, heart rate, right atrial diastolic pressure, respiratory frequency, axillary temperature, urine production, Glasgow coma scale score, presence or absence of jaundice; laboratory tests - blood workup, biochemical analysis, arterial blood gas analysis and fraction of inspired oxygen; use of vasopressor agents - dopamine, dobutamine, noradrenalin or adrenaline; prognostic scores acute physiology and chronic health evaluation (APACHE 11 and 111), simplified acute physiology score (SAPS 11), organ dysfunction and infection
(ODIN), multiple organ dysfunction score (MODS) and sepsis-related organ failure assessment (SOFA); evolution - number of days in the ICU and in the hospital, evolution in the $1 \mathrm{CU}$, evolution in the hospital, cause of death and the relation between death and infection.

The Bird 8400 ventilator with microprocessor (Bird Products Corp. Palm Springs, CA, USA) was used for $97.8 \%$ of the patients. For in-hospital transportation of the patients, T-Bird and Bird Mark-7 ventilators (Bird Products Corp.), as well as a NewPort ventilator (Newport Medical Instruments, Newport Beach, CA, USA), were used.

This being an observational study, no therapeutic strategy was devised or modified based on this cohort.

Together with the history and clinical presentation of the patient, the following criteria were used in the diagnosis of VAP: leukocyte counts greater than $10,000 / \mathrm{mm} 3$ or lower than $4000 / \mathrm{mm} 3$; axillary temperature $>38^{\circ} \mathrm{C}$ or $<36^{\circ} \mathrm{C}$; tracheal fluid that is purulent or presents an altered aspect; presence of new, persistent or progressive infiltrates, consolidations, pulmonary cavitations or pleural effusion (on the chest X-ray, computed tomography of the chest or both) - other noninfectious causes having been ruled out; and positivity in sample cultures of tracheal fluid aspirate, of bronchoalveolar lavage fluid (BALF) or both, as well as in blood samples, when available.

The VAP rates were described in accordance with the standard established by the National Control System of Nosocomial Infection of the Centers for Disease Control and Prevention (rate $=$ no. of VAP cases/1000 MV days). ${ }^{(16)}$ The microbiological diagnosis was performed through quantitative cultures of the BALF and tracheal fluid aspirate, as well as through blood cultures and lung biopsies. The following complications were also reported during MV: acute respiratory distress syndrome (ARDS); tracheobronchitis; acute sinusitis; atelectasis; pneumothorax; pneumomediastinum; and accidental extubation.

The data were entered into an electronic spreadsheet and presented as percentages (\%) or medians (interquartile range, 25\% - 75\%). For the categorical variables, the chi-square test was used (with Yates correction when indicated), and the Mann-Whitney Rank Sum test was used to compare the nonparametric continuous variables. To 
determine the correlation between two variables, we used linear regression and Pearson's correlation coefficient, as in the case of the variables days on $M V$ and percentage of pneumonia. For the multivariate analysis (binary logistic regression), we used the statistical software program Statistical Package for Social Sciences, version 10.0 (SPSS Inc., Chicago, IL, USA). The variables that reached $p<0.25$ were selected for logistic regression. The continuous variables were categorized, and the best cutoff points were selected by statistical determination. The selected variables are presented in tables with the respective coefficients (with standard errors) and odds ratios (with 95\% confidence intervals). After logistic regression, the discriminatory power and calibration of the models created were evaluated by calculating the area under the receiver operating characteristic curve and the Hosmer-Lemeshow goodness-of-fit statistic, respectively. In all cases, values of $p<$ 0.05 were considered significant.

Due to the merely observational and epidemiological character of the present study, written informed consent was not required. The study design was approved by the local ethics in research committee.

\section{RESULTS}

Over the space of eighteen months, 808 were hospitalized, 755 of which were admitted to the ICU. A total of 477 patients were excluded from the study because they did not meet the inclusion criteria. Therefore, 278 patients were included. The mean age was 52 years $(25 \%-75 \%$ interquartile range, 37 to 66 years), and 50\% of the patients were male.

Upon admission, 220 patients (79.1\%) were already intubated or had been submitted to tracheostomy, and 218 (78.4\%) were on MV. There were 150 patients (54\%) who presented respiratory insufficiency upon admission, and 92 (33.1\%) were diagnosed with pneumonia: 53 (57.6\% of the 92) with nosocomial pneumonia and 39 (42.4\% of the 92) with community-acquired pneumonia (prior to admission to the ICU). In addition to the patients submitted to tracheostomy prior to admission to the ICU, 73 (26.3\%) were submitted to tracheostomy during their stay in the ICU. On average, the procedure was carried out on MV day 11.1 (range, 7 to 16 days). None of the patients required or presented any condition that would necessitate the use of a nasotracheal tube.

Most (147) of the 1CU admissions (53\%) were for clinical reasons. A total of 76 patients (27\%) were admitted after an elective surgical procedure, and $55(20 \%)$ were admitted after an emergency surgical procedure. Thee were 30 patients (10.8\%) who had previously been admitted to the ICU.

The most common comorbidities were as follows: chronic obstructive pulmonary disease or restrictive lung disease (40/278 - 14.4\%); liver cirrhosis (33/278 $11.9 \%)$; chronic renal failure (25/278 - 9\%); congestive heart failure (21/278 - 7.6\%); immunosuppression (19/ 278 - 6.8\%); metastatic neoplasia (12/278 - 4.3\%); acquired immunodeficiency syndrome (9/278 - 3.2\%); and lymphoma or myeloma (9/278 - 3.2\%). The most common acute diseases were as follows: acute renal failure (44/278 - 15.8\%); cardiorespiratory arrest (28/ $278-10.1 \%)$, cardiac arrhythmia (23/278 - 8.3\%), upper gastrointestinal bleeding $(14 / 278-5 \%)$ and intracranial mass effect $(4 / 278-1.4 \%)$.

Within $24 \mathrm{~h}$ after admission, 149 patients (53.6\%) presented some confirmed infection, 235 (84.5\%) were on MV, $92(33.1 \%)$ required vasopressors for more than $1 \mathrm{~h}$, and $29(10.4 \%)$ were in a coma (Glasgow 5).

There were 106 patients (38.1\%) who developed VAP within a mean of four MV days (25\% - 75\% interquartile range, 2 to 19 days), translating to 35.7 cases/1000 MV days. Figure 1 presents the prevalence of VAP in relation to the number of MV days. The mean MV duration was 7 days ( 3 to 15 days). Among the patients who developed VAP, time on MV ranged from 2 to 93 days, with a median of 13 days $(25 \%-75 \%$ interquartile range, 7 to 20 days). In the group

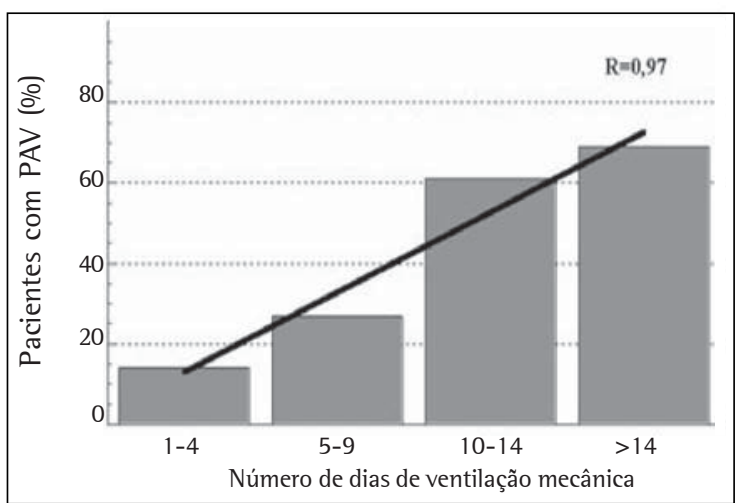

Figure 1 - Prevalence of VAP related to the number of days on mechanical ventilation 
without VAP, the time on MV was shorter, ranging from 1 to 37 days, with a median of 5 days (25\% - 75\% interquartile range, 2 to 9 days; $p<0.0001$ versus the VAP group).

A total of 69 patients with VAP (65.1\%) were admitted to ICU based on clinical causes, whereas the others were surgical patients.

The etiological agent was identified in 64 $(60.4 \%)$ of the patients with VAP, $29(45.3 \%)$ of whom were found to be infected with gram-negative agents. The bacteria most often isolated were Pseudomona aeruginosa (22\%), Acinetobacter spp. (14\%) and methicillin-resistant Staphylococcus aureus (11\%). The blood culture tested positive in $13.2 \%$ of the cases, and Staphylococcus spp was isolated in 43.7\% of those cultures. Most cultures $(28.6 \%)$ were polymicrobial, with equal frequency between the tracheal fluid aspirate and the BALF. Multidrug-resistant organisms were identified in 46 patients (43.4\%).

Although ARDS and infections caused by multidrug-resistant organisms do not result directly from MV, they are both included Table 1, in order to compare the groups of patients with and without VAP who developed complications while on MV. Re-intubation was necessary in 27 (13\%) of the patients, and $56(20.1 \%)$ required tracheostomy in order to be weaned from the ventilator.

There was no difference between the patients with and without VAP regarding the probability of death generated by the prognostic scores on the APACHE 11 and 111, as well as on the SAPS 11: the

\section{TABLE 1}

Complications presented by the patients during ventilatory support: comparison between the groups with and without VAP

\begin{tabular}{lcrll}
\hline & $\begin{array}{c}\text { WithPAV }(\%) \\
(\mathrm{n}=106)\end{array}$ & \multicolumn{2}{c}{ WithoutPAV $(\%)$} & $p$ \\
$(\mathrm{n}=172)$ & & \\
\hline ARDS & $23(21.7)$ & $11(6.4)$ & $<0.001$ \\
Tracheobronchitis & $10(9.4)$ & 5 & $(2.9)$ & $<0.05$ \\
Sinusitis & $12(11.3)$ & 1 & $(0.6)$ & $<0.001$ \\
Atelectasis & $24(22.6)$ & 11 & $(6.4)$ & $<0.001$ \\
Pneumothorax & $14(13.2)$ & $9(5.2)$ & $<0.05$ \\
MRl & $46(43.4)$ & $21(12.2)$ & $<0.001$ \\
Pneumomediastinum & $2(1.9)$ & 0 & $(0)$ & NS \\
Accidental extubation & $18(17)$ & 16 & $(9.3)$ & NS \\
\hline ARDS:
\end{tabular}

ARDS: acute respiratory distress syndrome; VAP: ventilatorassociated pneumonia; MRl: multidrug-resistant infection; NS: not significant.
APACHE 11 score was 38.6\% (21\% to 66.9\%) for the patients with VAP, compared with $39.7 \%$ $(17.3 \%$ to $63.6 \%)$ for those without $(p=$ not significant); the APACHE 111 score was 74.5 points (59 to 95 points) for the patients with VAP, compared with 73 points (54 to 91 points) for those without ( $p=$ not significant); The SAPS 11 was $30.6 \%$ ( $12.8 \%$ to $57.5 \%)$ for the patients with VAP, compared with $28.5 \%$ ( $10.2 \%$ to $58.6 \%)$ for those without ( $p=$ not significant). However, there were differences among the organ dysfunction scores (MODS, SOFA and ODIN): the MODS was 7 points (5 to 10 points) for the patients with VAP, compared with 6 points (4 to 8 points) for those without ( $p$ $<0.05$ ); the SOFA score was 9 points (6 to 12 points) for the patients with VAP, compared with 8 points (5 to 11 points) for those without ( $p<$ $0.05)$; the ODIN score was $21.3 \%$ (12.2\% to $31.4 \%)$ for the patients with VAP, compared with $12.2 \%$ $(7.59 \%-31.4 \%)$ for those without $(p<0.05)$.

Table 2 shows the time on MV, time to MV weaning, length of ICU stay, length of hospital stay, patient evolution in the ICU and patient evolution in the hospital. In the patients with VAP, time on MV, time to MV weaning, length of ICU stay and length of hospital stay were increased. However, this did not result in higher mortality rates in the ICU or in the hospital.

Table 3 shows VAP-related factors, selected by binary logistic regression. We observed that the variables acute sinusitis, atelectasis, ARDS, immunosuppression and more than ten days on MV

\section{TABLE 2}

Time on mechanical ventilation; mechanical ventilation weaning; length of ICU and hospital stays [in days - median (25-75\% interquartile range)]; and mortality in-1CU and in-hospital (number/ total - percentage) among the 278 patients admitted to the ICU and in the groups with and without VAP

\begin{tabular}{lccrr}
\hline & $\begin{array}{c}\text { Total } \\
(\mathrm{n}=278)\end{array}$ & $\begin{array}{c}\text { With PAV } \\
(\mathrm{n}=106)\end{array}$ & $\begin{array}{c}\text { Without PAV } \\
(\mathrm{n}=172)\end{array}$ & $p$ \\
\hline Mechanical ventilation & $7(3-15)$ & $13(7-20)$ & $5(2-9)$ & $<0,001$ \\
MV weaning & $2(1-4)$ & $4(2-8)$ & $1(1-3)$ & $<0,001$ \\
Length of 1CU stay & $8(3-15)$ & $14(9-22)$ & $5(3-10)$ & $<0,001$ \\
Length of stay in hospital & $25(12-46)$ & $35(15-53)$ & $21(10-42)$ & $<0,001$ \\
Mortality in the 1CU 126/278 (45.3\%) & $47 / 106(44.3 \%)$ & $79 / 172(45.9 \%)$ & NS \\
ln-hospital mortality 155/278 (55.8\%) & $63 / 106(59.4 \%)$ & $92 / 172(53.5 \%)$ & NS \\
\hline
\end{tabular}

MV: mechanical ventilation; VAP: ventilator-associated pneumonia; ICU: intensive care unit; NS: not significant 
TABLE 3

Factors, selected through binary logistic regression, related to the presence of VAP in patients on mechanical ventilation admitted to the $\mathrm{CCU}$

\begin{tabular}{lccr}
\hline Variable & $\begin{array}{c}\text { Coefficient } \\
+ \text { erro padrão }\end{array}$ & \multicolumn{1}{c}{$\begin{array}{c}\text { Odds Ratio } \\
(1 \mathrm{C} \mathrm{95 \% )}\end{array}$} & \multicolumn{1}{c}{$\mathrm{p}$} \\
\hline Acute sinusitis $(\mathrm{Y} / \mathrm{N})^{*}$ & $3.658+1.240$ & $38.794(3.413-440.985)$ & 0.03 \\
$>$ 10 days on $\mathrm{MV}(\mathrm{Y} / \mathrm{N})$ & $2.038+0.315$ & $7.672(4.138-14.222)$ & $<0.001$ \\
lmmunosuppression $(\mathrm{Y} / \mathrm{N} 1.456+0.616$ & $4.290(1.283-14.342)$ & 0.009 \\
ARDS $(\mathrm{Y} / \mathrm{N})$ & $1.252+0.482$ & $3.497(1.360-8.995)$ & 0.009 \\
Atelectasis $(\mathrm{Y} / \mathrm{N})$ & $1.090+0.459$ & $2.973(1.208-7.314)$ & 0.018 \\
CA $(\mathrm{Y} / \mathrm{N})$ & $-1.730+0.667$ & $0.177(0.048-0.655)$ & 0.009 \\
UGlB $(\mathrm{Y} / \mathrm{N})$ & $-2.612+1.086$ & $0.073(0.009-0.617)$ & 0.016 \\
Constant & -1.690 & & \\
\hline
\end{tabular}

Discriminatory power: Area under the receiver operating characteristic curve, $0.836 \pm$ 0.025 (95\% Cl: 0.786-0.886); calibration: goodness-of-fit test, $\mathrm{C}^{\wedge}=0.661(\mathrm{p}=0.985)$; VAP: ventilator-associated pneumonia; MV: mechanical ventilation; ARDS: acute respiratory distress syndrome; CA: Cardiac arrest; UGIB - upper gastrointestinal bleeding; 95\% Cl: 95\% confidence interval; ${ }^{*} \mathrm{Y}$ (yes) $=1$, and $\mathrm{N}($ no $)=0$

presented positive associations with VAP. However, the variables upper gastrointestinal bleeding and cardiorespiratory arrest were found to be protective, since the incidence of VAP was lower among the patients who presented these characteristics. The model presented good discriminatory power (area under the receiver operating characteristic curve $=0.836 \pm 0.025$; 95\% confidence interval, $0.786-0.886)$ and good calibration (goodness-of-fit test, $\left.C^{\wedge}=0.661\right)(p=0.985)$.

Table 4 shows the variables found to be associated with in-hospital death, which were selected by binary logistic regression. We observed that chronic renal failure, previous ICU admission (during the same hospitalization), being over the

\section{TABLE 4}

Factors, selected by binary logistic regression, related to inhospital death among patients with VAP

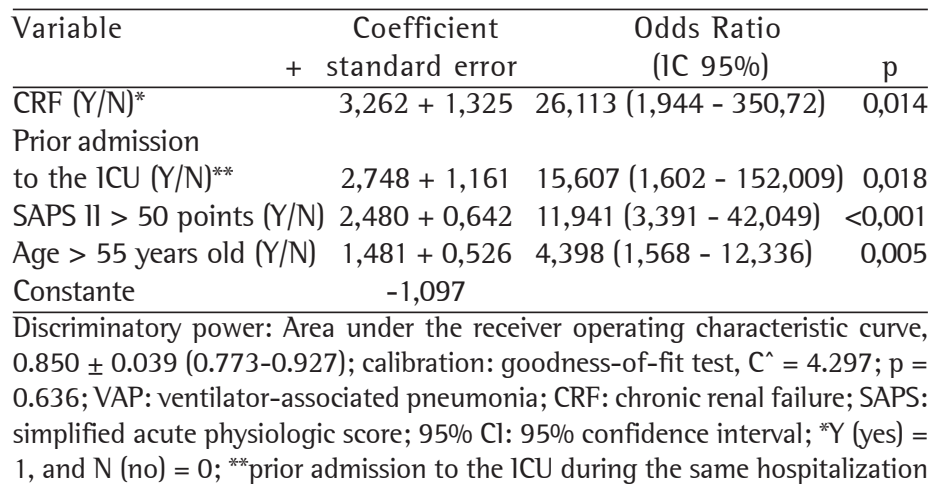

age of 55 and SAPS $11>50$ points were positively associated with in-hospital death. The model also presented good discrimination (area under the receiver operating characteristic curve, $0.850 \pm$ 0.039; 95\% confidence interval: 0.773 - 0.927); and good calibration (goodness-of-fit test, $\mathrm{C}^{\wedge}=$ 4297; $p=0.636)$.

\section{DISCUSSION}

In the present study, $38.1 \%$ of the evaluated patients developed VAP, translating to 35.7 cases/ 1000 MV days. Quite diverse rates have been found, from 20.5 to 34.4 cases/1000 MV days. ${ }^{(9-10,12)}$ These results surpass the data of the National Nosocomial Infections Surveillance System, ${ }^{(13)}$ which reports a rate of 13.5 cases/1000 MV days. There is currently no gold standard test for VAP diagnosis and no specific method for ruling out pulmonary infection in patients on MV with fever and multiple organ dysfunction syndrome. This may be the reason why VAP rates vary so greatly among units, hospitals, regions and countries. Even postmortem histological diagnostic studies are inconclusive. ${ }^{(14)}$ The dosage of soluble triggering receptor expressed on myeloid cells- 1 in BALF has been evaluated as a rapid-execution, fast-result diagnostic test for pneumonia. ${ }^{(15)}$

For example, if we consider the results of tracheal fluid cultures, the incidence of VAP can be twice as high as in those cases in which the diagnosis is made only through the use of BALF.(5) In the present study, both techniques of sample collection were used, with approximately 60\% positivity in all the cultures we conducted. In contrast to various studies that select patients based on the type of examination used in the diagnosis of VAP (generally BALF), our observational study did not and is therefore closer to the reality of current medical practice. Regarding the etiologic diagnosis, we observed concordance with the agents typically isolated in late-onset VAP. ${ }^{(16-17)}$

In the present study, 92 cases of late-onset VAP (87\%) occurred. A total of $43 \%$ of the patients with VAP presented infection with multidrugresistant organisms, a statistically higher value than that found for the group without VAP $(p<0.001)$.

In analyzing the differences between the two groups in terms of complications during MV, we observed that the patients with VAP presented 
ARDS, pneumothorax, atelectasis, tracheobronchitis and sinusitis more frequently than did those in the group without VAP, all of the differences being statistically significant (Table 2). The sinuses can be reservoirs of nosocomial pathogens, contributing to the colonization of the oropharynx.$^{(7)}$ Regarding ARDS, we recommend an increased level of clinical suspicion of pneumonia. Moreover, the presence of only one of the clinical criteria (fever, leukocytosis or purulent tracheal fluid), hemodynamic instability or hypoxemia should motivate a search for a diagnosis of VAP through the collection of cultures and through diagnostic imaging (such as computed tomography of the chest). ${ }^{(18)}$

As can be seen in Table 2, 126 patients died in the ICU. Of those 126 deaths, 78 (62\%) were related to the infection, VAP or not. This means that death from infection is still a serious problem and that, in the group with VAP, the organ dysfunction scales (MODS, SOFA and ODIN), which evaluate infection and sepsis, presented good performance in the study.

The patients on MV presented high mortality rates. These already high mortality rates were not influenced by the presence or absence of VAP, either in the ICU or in the hospital. Some authors ${ }^{(19)}$ have observed higher mortality rates in the patients with VAP. However, when the impact that VAP had on the time on MV, time to weaning, length of ICU stay and length of hospital stay was analyzed in the present study, the values were quite distinct $(p<0.001)$ (Table 2). Other authors ${ }^{(12,20,22)}$ have suggested that the severity of the clinical profile of the patients at the moment of the VAP diagnosis is the most important factor affecting survival in the ICU.

Through the multivariate analysis, we observed that the presence of acute sinusitis was strongly associated with VAP, but with a wide confidence interval due to the small number of cases (Table 3). Atelectasis was also a factor strongly associated with VAP, probably due to the greater use of MV in these patients. The time on MV is a well-recognized factor that is strongly associated factor with the development of nosocomial pneumonia. In the present study, the majority of cases were late-onset VAP. Therefore, the time on MV of over ten days was the variable identified as having the second leading odds ratio for VAP, which confirms the findings of other studies. ${ }^{(19)}$ Immunosuppression was another VAP-related factor, despite the small number of cases in our sample (6.8\%). If we take into consideration the conditions of metastatic neoplasm, lymphoma, myeloma, and acquired immunodeficiency syndrome, the number of "immunosuppressed" individuals increases (49/ 278). The greater susceptibility to infection among these patients is justified by their impaired immunological state, a factor strongly associated with VAP. Patients with ARDS are also predisposed to pulmonary infection (within $24 \mathrm{~h}$ of an ARDS diagnosis) between $34 \%$ and $70 \%$ of the cases, frequently leading to sepsis, multiple organ dysfunction syndrome and death. ${ }^{(3)}$ Some authors ${ }^{(21)}$ found evidence of pneumonia in samples of lung biopsies in 73\% of the cases of patients with ARDS. Cardiac arrest prior to admission to the ICU was identified as a factor inversely related to VAP. Of the 28 patients who had presented cardiac arrest prior to admission to the 1CU, only 7 developed VAP (25\%). Nineteen (68\%) of these patients died, including 5 of those who developed VAP. Therefore, many patients died before developing VAP. Upper gastrointestinal bleeding was also a factor identified through logistic regression as being inversely related to the incidence of VAP. The patients with upper gastrointestinal bleeding might have presented a gastric $\mathrm{pH}$ that was more acid. It is well known that the inhibition of the gastric secretion with histamine type 2 receptor blockers or with proton pump inhibitors or enteral feeding favors the occurrence of VAP. ${ }^{(19)}$

The difference between the patients with and without VAP in terms of the SAPS was not statistically significant. However, a high SAPS (>50 points) was associated with in-hospital mortality among the patients with VAP. Age and the presence of chronic renal failure were also associated with in-hospital death, demonstrating the impairment of the functional state that accompanies age and the presence of chronic diseases in patients with VAP. ${ }^{(22)}$ Age and chronic diseases are frequently related with the prognosis. A recent article revealed that mortality is greater in elderly patients with VAP. ${ }^{(23)}$ The authors of another article stated that the comorbidities decrease the chances of survival after admission to the 1CU. ${ }^{(24)}$

A recent multicenter study that analyzed critically ill patients with chronic renal failure and the prognostic impact, found a $60.3 \%$ mortality rate for these patients. The greatest independent 
risk factors for in-hospital mortality were MV and the use of vasopressor agents. ${ }^{(25)} \mathrm{In}$ identifying the characteristics of the patients with chronic renal failure in the various health facilities, it was observed that approximately $30 \%$ presented chronic renal damage (premorbid renal dysfunction). It is thus understood that chronic disease has a strong impact in patients with infection who develop sepsis, septic shock or organic dysfunction, worsening the inhospital prognosis.

Prior admission to the ICU can correspond to a scenario of ever more frequent chronicity, demonstrating worse prognosis in the long run, according to which the patients no longer meet the requirements for being discharged, develop a chronic state and are referred to the ICU, and that is when they suffer an acute event.

The present study was carried out in a single ICU in a university hospital in the city of Rio de Janeiro. Therefore, we recommend that extreme caution be taken in extrapolating the data presented here to other facilities in other hospitals. Different MV practices, diagnostic criteria, and methods of bacteriological diagnosis can modify the results reported here.

This study determined the prevalence of VAP in the ICU of the Clementino Fraga Filho University Hospital of the Federal University of Rio de Janeiro, identifying the associated factors and the inhospital prognosis. Knowledge of these factors is important to elucidating the questions of the professionals who deal with these critically ill patients and their families. Some of these factors can be avoided, which would decrease VAP incidence VAP-related mortality.

\section{ACKNOWLEDGMENTS}

We are extremely grateful to Professor Rocco for his dedication to and enthusiasm for our academic development, as well as for his scientific encouragement that we were able to throughout our years in medical school, medical residence and postgraduate studies at the Federal University of Rio de Janeiro. We also wish to thank the entire medical team of the Intensive Care Unit of the Center for Surgery and Internal Medicine at the Clementino Fraga Filho University Hospital. In addition, we thank Dr. Marco Aurélio Albuquerque for inspiring higher standards in the quality of medical treatment and in the pursuit of scientific knowledge.

\section{REFERENCES}

1. McEachern R, Campbell GD Jr. Lower respiratory tract infections: Hospital-acquired pneumonia: epidemiology, etiology and treatment. Infec Dis Clin North Am. 1998;12(3):761-79.

2. Lode H, Raffenberg M, Erbes R, Geerdes-Fenge H, Mauch H. Nosocomial pneumonia: epidemiology, pathogenesis, diagnosis, treatment and prevention. Curr Opin Infect Dis. 2000;13(4):377-84.

3. Chastre J, Fagon JY. Ventilator-associated Pneumonia. Am J Respir Crit Care Med. 2002;165(7):867-903.

4. Richards MJ, Edwards JR, Culver DH, Gaynes RP. Nosocomial infections in medical intensive care units in the United States. Crit Care Med. 1999;27(5):887-92.

5. American Thoracic Society; Infectious Diseases Society of America. Guidelines for the management of adults with hospital-acquired, ventilator-associated, and healthcare-associated pneumonia. Am J Resp Crit Care Med. 2005;171(4):388-416.

6. Craven DE, Steger KA. Epidemiology of nosocomial pneumonia: new perspective on an old disease. Chest. 1995;108(2 Suppl):1S-16S.

7. Rouby JJ, Laurent P, Gosnach M, Cambau E, Lamas G, Zouaoui A, et al. Risk factor and clinical relevance of nosocomial maxillary sinusitis in the critically ill. Am J Respir Crit Care Med. 1994;150(3):776-83.

8. Tablan OC, Anderson LJ, Arden NH, Breiman RF, Butler JC, McNeil MM. Guidelines for prevention of nosocomial pneumonia. The Hospital Infection Control Practices Advisory Committee, Centers for Disease Control and Prevention. Am J Infect Control. 1994;22(4):587-627.

9. George DL, Falk PS, Wunderink RG, Leeper KV Jr, Meduri GU, Steere EL, et al. Epidemiology of ventilator-acquired pneumonia based on protected bronchoscopic sampling. Am J Respir Crit Care Med. 1998;158(6):1839-47.

10. Fagon JY, Chastre J, Domart Y, Trouillet JL, Pierre J, Darne $\mathrm{C}$, et al. Nosocomial pneumonia in patients receiving continuous mechanical ventilation. Prospective analysis of 52 episodes with the use of a protected specimen brush and quantitative culture techniques. Am Rev Resp Dis. $1989 ; 139(4): 877-84$.

11. Skerrett SJ. Pneumonia: overview and approach to diagnosis and management. In: Albert RK, Dries DJ, eds. ACCP/SCCM Combined Critical Care Course - Multidisciplinary Board Review. United States of America: Society of Critical Care Medicine and the American College of Chest Physicians. Editors. 2000:163-78.

12. Woske HJ, Roding T, Schulz 1, Lode H. Ventilatorassociated pneumonia in a surgical intensive care unit: epidemiology, etiology and comparison of three bronchoscopic methods for microbiological specimen sampling. Critical Care. 2001;5(3):167-73.

13. National Nosocomial Infections Surveillance (NN1S) System Report: data summary from January 1992-April 2000, issued June 2000. Am J Infect Control. 2000;28(6):429-48. 
14. Corley DE, Kirtland SH, Winterbauer RH, Hammar SP, Dail DH, Bauermeister DE, et al. Reproducibility of the histologic diagnosis of pneumonia among a panel of four pathologists: analysis of a gold standard. Chest. 1997;112(2):458-65.

15. Gibot S, Cravoisy A, Levy B, Bene MC, Faure G, Bollaert PE. Soluble triggering receptor expressed on myeloid cells and the diagnosis of pneumonia. $\mathrm{N}$ Engl $\mathrm{J}$ Med. 2004;350(5):451-8.

16. Timsit JF, Chevret S, Valcke J, Misset B, Renaud B, Goldstein FW, et al. Mortality of nosocomial pneumonia in ventilated patients: influence of diagnostic tools. Am J Respir Crit Care Med. 1996;154(1):116-23.

17. Torres A, Carlet J. Ventilator-associated pneumonia. European Task Force on ventilator-associated pneumonia. Eur Respir J. 2001;17(5):1034-45

18. Delclaux C, Roupie E, Blot F, Brochard L, Lemaire F, Brun-Buisson C. Lower respiratory tract colonization and infection during severe acute respiratory distress syndrome: incidence and diagnosis. Am J Respir Crit Care Med. 1997;156(4 Pt 1):1092-98.

19. Cook DJ, Walter SD, Cook RJ, Griffith LE, Guyatt GH, Leasa $D$, et al. Incidence of and risk factors for ventilator-associated pneumonia in critically ill patients. Ann Inter Med. 1998;129(6):433-40.

20. Rello J, Rue M, Jubert P, Muses G, Sonora R, Valles J, et al. Survival in patients with nosocomial pneumonia: impact of the severity of illness and the etiologic agent. Crit Care Med. 1997;25(11):1862-7.

21. Bell RC, Coalson JJ, Smith JD, Johanson WG Jr. Multiple organ system failure and infection in adult respiratory distress syndrome. Ann Intern Med. 1983;99(3):293-8.

22. Bregeon F, Ciais V, Carret V, Gregoire R, Saux P, Gainnier $M$, et al. Is ventilator-associated pneumonia an independent risk factor for death? Anesthesiology. 2001;94(4):554-60.

23. Janssens JP, Krause KH. Pneumonia in the very old. Lancet Infect Dis. 2004;4(2):112-24.

24. Hamel MB, Davis RB, Teno JM, Knaus WA, Lynn J, Harrell F Jr, et al. Older age, aggressiveness of care, and survival for seriously ill, hospitalized adults. Ann Intern Med. 1999;131(10):721-8.

25. Uchino S, Kellum JA, Bellomo R, Doig GS, Morimatsu $\mathrm{H}$, Morgera S, et al. Acute renal failure in critically ill patients. A multinational, multicenter study. JAMA. 2005;294(7):813-8. 